\title{
Sperm survival in the female reproductive tract in the fly Scathophaga stercoraria (L.)
}

\author{
G. Bernasconi ${ }^{\mathrm{a}, *}$, B. Hellriegel ${ }^{\mathrm{b}}$, A. Heyland ${ }^{1, \mathrm{~b}}$, P.I. Ward ${ }^{\mathrm{b}}$ \\ a Institute of Environmental Sciences, University of Zurich, Winterthurerstr. 190, 8057 Zurich, Switzerland \\ ${ }^{\mathrm{b}}$ Zoological Museum, University of Zurich, Winterthurerstr. 190, 8057 Zurich, Switzerland
}

Received 7 August 2001; received in revised form 19 October 2001; accepted 22 October 2001

\begin{abstract}
While sperm competition risk favours males transferring many sperm to secure fertilizations, females of a variety of species actively reduce sperm numbers reaching their reproductive tract, e.g. by extrusion or killing. Potential benefits of spermicide to females include nutritional gains, influence over sperm storage and paternity, and the elimination of sperm bearing somatic mutations that would lower zygote fitness.

We investigated changes in sperm viability after in vivo and in vitro exposure to the female tract in the polyandrous fly, Scathophaga stercoraria. Sperm viability was significantly lower in the females' spermathecae immediately after mating than in the experimental males' testes. Males also varied significantly in the proportion of live sperm found in storage in vivo. However, the exact mechanism of sperm degradation remains to be clarified. In vitro exposure to extracts of the female reproductive tract, including female accessory glands, failed to significantly lower sperm viability compared to controls. These results are consistent either with postcopulatory sperm mortality in vivo depending entirely on the male (with individual differences in sperm viability, motility or longevity) or with postcopulatory sperm mortality being subtly affected by female effects which were not detected by the in vitro experimental conditions. Importantly, we found no evidence in support of the hypothesis that female accessory glands contribute to sexual conflict via spermicide. Therefore, female muscular control remains to date the only ascertained mechanism of female influence on sperm storage in this species. (C) 2002 Elsevier Science Ltd. All rights reserved.
\end{abstract}

Keywords: Female reproductive tract; Sperm storage; Sperm viability; Spermicide; Sexual conflict; Diptera; Female accessory gland; Scatophaga

\section{Introduction}

In many taxa the number of sperm stored by females is much lower than that transferred by males at mating. For instance, in the beetles Tribolium castaneum (Bloch Qazi et al., 1996) and Callosobruchus maculatus (Eady, 1994), only $4 \%$ and $15 \%$, respectively, of the sperm transferred reach the spermatheca. Females of some species directly extrude sperm after mating (e.g. carrion flies, Otronen and Siva-Jothy, 1991; millipedes, Barnett et al., 1995; feral fowl, Pizzari and Birkhead, 2000).

\footnotetext{
* Corresponding author. Tel.: +41-1-635-4807; fax: +41-1-6355711.

E-mail addresses: bernasco@uwinst.unizh.ch (G. Bernasconi), barhell@zool.unizh.ch (B. Hellriegel), aheyland@zoo.ufl.edu (A. Heyland), pward@zooolmus.unizh.ch (P.I. Ward).

${ }_{1}^{1}$ Present address: Department of Zoology, University of Florida, 223 Bartram Hall, POB 118525, Gainesville, FL 32611-8525, USA.
}

Moreover, the female reproductive tract can be hostile to sperm (Birkhead et al., 1993), reducing the viability and/or fertilization ability of sperm reaching storage, as suggested by evidence of sperm digestion starting immediately after sperm transfer at copulation (flatworms, Michiels and Bakovski, 2000), sperm degradation in the bursa copulatrix (bruchid beetles, Eady, 1994), and that female accessory gland secretions debilitate spermatozoa (house fly, Degrugillier, 1985). Numerical and functional elimination of sperm prior to fertilization counters male interests because males are selected to respond to increased sperm competition risk by maximizing their representation in the fertilization set (Parker, 1990; Hellriegel and Ward, 1998).

Providing a hostile environment for sperm may allow females to (i) promote male competition (Birkhead et al., 1993; Keller and Reeve, 1995; Bernasconi and Keller, 2001), (ii) obtain nutritional benefits (Wickler, 1985; Arnqvist and Nilsson, 2000), (iii) eliminate sperm bear- 
ing somatic mutations (Jones et al., 2000; Siva-Jothy, 2000), (iv) counter antagonistic male adaptations, e.g. to neutralize male-derived substances with detrimental effects on female fitness (Chapman et al., 1995; Rice, 1996; Johnstone and Keller, 2000; Andrès and Arnqvist, 2000), or (v) influence paternity (Birkhead et al., 1993; Birkhead, 1998; Hellriegel and Ward, 1998; Greeff and Parker, 2000).

In the fly Scathophaga stercoraria, females have influence over paternity (Ward, 2000a). Theory suggests two mechanisms by means of which females can bias paternity: control of storage rates to multiple spermathecae, and spermicide (Hellriegel and Ward, 1998; Greeff and Parker, 2000). Evidence indicates that sperm storage rates depend on female muscular activity (Simmons et al., 1999), and this results in different proportions of sperm from a female's mates being stored across her multiple spermathecae (Hellriegel and Bernasconi, 2000). In contrast, it is not known whether spermicide occurs, nor are potential mechanisms and their quantitative effects identified. Female tract physiology and products have not been as extensively investigated in Diptera as male-derived products (e.g. Drosophila males, Chapman et al., 1995; Rice, 1996). In S. stercoraria, female reproductive accessory gland secretions are known to be released during copula (Hosken and Ward, 1999). The glands were found to be larger when females were mated to several males vs. one male after 10 generations (Hosken et al., 2001). Moreover, if sperm are exposed to a hostile environment prior to storage, males may differ with respect to their ability to resist sperm degradation in the female tract, providing a new predictor of variation in paternity success among males. Here we investigate sperm survival after exposure (i) to the female reproductive tract in vivo using virgin males of different age at first mating and (ii) to extracts of different regions of the female tract, including female accessory glands, in vitro.

\section{Material and methods}

We collected S. stercoraria mating pairs in the field (Fehraltdorf, Switzerland, November 1999) and raised their progeny in the laboratory (larvae: $14{ }^{\circ} \mathrm{C}$; adults: 19 ${ }^{\circ} \mathrm{C}$, light/dark cycle 13/11 hours, Ward and Simmons, 1991). After emergence, virgin flies were kept singly and supplied with water, sugar and Drosophila as prey.

\subsection{Sperm viability assessment}

We assessed sperm viability with the live/dead ${ }^{\mathrm{TM}}$ Sperm Viability Kit (L-7011, Molecular Probes), which consists of a membrane-permeant nucleic acid stain (SYBR14; diluted 1:50; emission max. $516 \mathrm{~nm}$ ) and a dead-cell stain (probidium iodide; emission max. 617 $\mathrm{nm}$ ). Both solutions stain sperm heads only (Fig. 1). We used Schneider's Medium (GIBCO BRL 21 720-024) with heat-inactivated $\left(30 \mathrm{~min} / 56{ }^{\circ} \mathrm{C}\right) \quad 10 \%$ fetal calf serum (EUROBIO 01 0056) as buffer. This protocol is an optimization of the protocol of Molecular Probes for our study organism. Samples were analyzed for epifluorescence (Reichert-Jung Polyvar microscope, Hamamatsu C5405, Argus-20). To ensure accuracy, we examined 46 images and recorded them under both light and fluorescence microscopy. Ninety-six percent of the cells seen in light microscopy could be found in the fluorescence image. Overall, 5\% (678 out of 13654 sperm cells) of the cells were doubly-stained (green in centre, red at ends), and were included as dead cells. In a preliminary experiment, we ensured that no dead sperm stain green by heating the testes $\left(10 \mathrm{~min} / 60^{\circ} \mathrm{C}\right)$, which resulted in all cells staining red. We counted live sperm (green, 485/10, BP 540/20) automatically (NIH Image), after calibration to sperm head size in a preliminary experiment (320 images, 4 males). Dead sperm (red, $557.5 / 27.5$ ) were counted manually, to avoid counting dead tissue of a size similar to sperm heads.

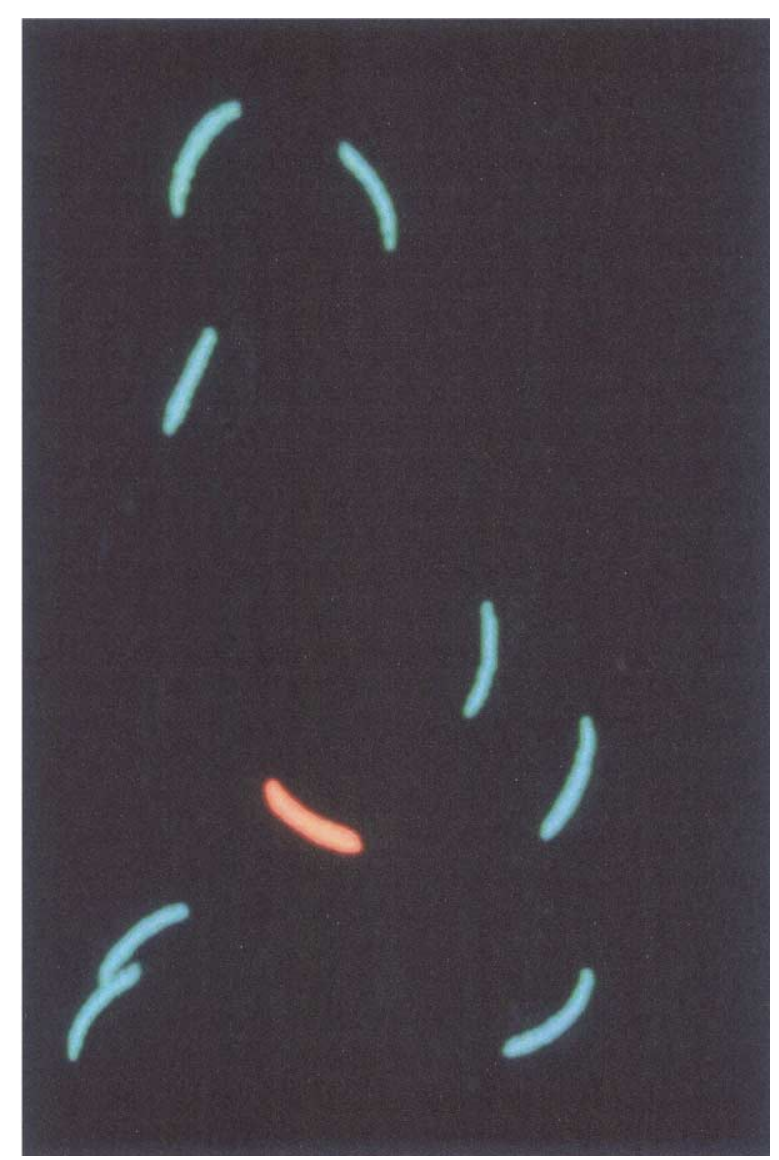

Fig. 1. Live sperm stain green (485/10, BP 540/20), dead sperm red (557.5/27.5; live/dead ${ }^{\mathrm{TM}}$ Sperm Viability Kit L-7011, Molecular Probes). 


\subsection{Sperm viability after in vivo exposure to the female tract}

We compared the proportion of live sperm in the spermathecae (i.e. after exposure to the female tract) to the proportion of live sperm in the males' testes for 20 mating pairs. Flies were dissected at the end of copulation (see Hellriegel and Bernasconi, 2000). Because male age could either affect sperm viability in the male or its ability to resist degradation within the female tract, in 10 mating pairs we used young males $(18 \pm 2$ days post-

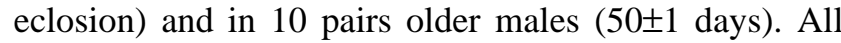
males were virgin, thus male age corresponds to age at first mating. The females' spermathecae were placed in $60 \mu \mathrm{l}$ buffer with $5 \mu \mathrm{l}$ of each stain, vortexed and transferred to a microslide in $40 \mu \mathrm{l}$. Sperm were released by exerting pressure on the cover glass (see control experiment). For the male, we opened each testis separately in buffer on a microslide and released the sperm from the third proximal to the ejaculatory duct. For each testis we added $5 \mu \mathrm{l}$ of each stain to $60 \mu \mathrm{l}$ of suspension, vortexed, and transferred $40 \mu \mathrm{l}$ to a microslide. We counted live/dead sperm on each of 20 (19-21) images for each testis and for the spermathecae. Images were recorded $20 \pm 6$ min after dissection and $9 \pm 4$ min after adding stains. Images had $35 \pm 23$ live and $7 \pm 11$ dead sperm; 57 out of 1197 (5\%) with tissue debris were excluded from analysis. Females were $36 \pm 6$ days old.

In addition, we carried out a control experiment to ensure that our procedure for preparing sperm from the testis vs. spermathecae did not affect sperm viability. We dissected both testes of each of six males and prepared two separate sperm suspensions which we stained as described for testis (see above). For one of the male's testes we transferred $40 \mu$ of stained suspension to a microslide and examined it directly. For the other testis, we transferred $40 \mu \mathrm{l}$ of stained suspension to a microslide on which we placed the spermathecae of a virgin female (i.e. containing no sperm) and exerted pressure on the cover glass until the spermathecal walls opened, ensuring comparable pressure as for preparing spermathecal sperm in the in vivo experiment. We examined $320 \pm 123$ sperm/male (72 images, 6 males).

\subsection{Sperm viability after in vitro after exposure to female reproductive tissue}

To identify whether, and which part of, the female tract might be responsible for sperm mortality, we exposed samples of sperm (from 24 males, 12 in each of two experimental blocks) to a set of suspensions containing buffer and isolated female reproductive tissues (female accessory glands, spermathecae, bursa copulatrix, bursa copulatrix with female accessory glands) or to a control (male ejaculatory bulb, male flight muscle, female flight muscle, buffer only). All suspensions were kept frozen $\left(-20^{\circ} \mathrm{C}, 6\right.$ days), and thawed and centrifuged (13 000g/2 min $/ 4{ }^{\circ} \mathrm{C}$; Haereus Biofuge Fresco) before use. Sperm obtained from virgin males $(n=24)$ were released in $100 \mu$ l buffer. We incubated ( \pm SD: $11 \pm 2 \mathrm{~min}$, room temperature) $15 \mu \mathrm{l}$ sperm suspension with $30 \mu \mathrm{l}$ buffer and $15 \mu \mathrm{l}$ tissue suspension. After incubation, we added $5 \mu$ of each stain, vortexed and immediately examined $30 \mu \mathrm{l}$ of the mixture. We recorded $108 \mathrm{images} / \mathrm{male}, 5 \pm 1 \mathrm{~min}$ after staining. Each image had $17 \pm 10$ live and $4 \pm 3$ dead sperm in the first and $13 \pm 8$ live sperm and $3 \pm 3$ dead sperm in the second block. Results of ANOVA adjusted for covariates (time from testis dissection, $p=0.64$; duration of incubation, $p=0.11$ ) were consistent with the analysis without covariates.

The temporal sequence of all procedures was randomized, sperm counts and incubation sequence were blind. All dissections were under $\mathrm{CO}_{2}$. Unless specified, data are given as mean \pm SD. Angularly transformed viabilities were analyzed using GENSTAT 5.3.2 (1995, Lawes Agricultural Trust, Rothamsted Experimental Station) with appropriate $F$-tests.

\section{Results}

The proportion of live sperm in the spermathecae immediately after mating and exposure to the female tract was significantly lower than in the male's testes (Table $1, F_{1,18}=12.98, p=0.002$, Figs. 2 and 3 ). In the spermathecae, viability was $79.2 \pm 10.3 \%$ (range $62.2-$ $95.3 \%$ ), in the testis dissected first it was $88.6 \pm 4.7 \%$ $(80.2-97.1 \%)$ and in the testis dissected last $82.7 \pm 3.7 \%$ (82.7-94.2\%). The difference between sperm viability in the spermathecae and the average sperm viability of the two testes was $9.8 \pm 11.4 \%$, with the most extreme difference for a given male being $30.1 \%$ lower viability in the spermathecae. These results indicate that sperm can die very soon after insemination.

Sperm viability did not differ significantly between a male's testes (Table $1, F_{1,18}=0.39, p=0.54$ ), indicating that there was no significant overall difference in the proportion of live sperm between the testis dissected first and second after a single mating (when averaged, the slope of the lines connecting individual values of sperm viability in testis 1 and testis 2 in Fig. 3 is flat). That there was no significant difference between the testis dissected first and second ensures that the procedure to assess sperm viability was rapid enough to avoid artifacts. Sperm survival through storage processes, i.e. the difference between sperm viability in the testes and in the spermathecae, varied widely and significantly among males $\left(F_{18,1080}=15.56, p<0.001\right.$, Fig. 3). However, this variation was not significantly explained by the age at which males were allowed their first mating (young: $18 \pm 2$ days post-emergence, $n=10$; old: $50 \pm 1$ days, $n=10$, 
Table 1

Accumulated analysis of variance for male age at first mating and sperm source (testes or spermathecae) on angularly-transformed sperm viability in vivo

\begin{tabular}{|c|c|c|c|c|c|c|}
\hline Source of variation & df & & ss & $\mathrm{ms}$ & $F_{\text {obs }}$ & $p\left(F>\left|F_{\text {obs }}\right|\right)$ \\
\hline Male age & 1 & & 0.04 & 0.04 & 0.05 & 0.83 \\
\hline Latency to copulation & 1 & & 2.12 & 2.12 & 2.30 & 0.15 \\
\hline Copulation duration & 1 & & 0.03 & 0.03 & 0.03 & 0.86 \\
\hline Residual among males & 16 & & 14.7 & 0.92 & & \\
\hline Source of sperm & 2 & & 22.4 & 22.4 & 11.17 & $<0.001$ \\
\hline \multicolumn{7}{|l|}{ Planned contrasts } \\
\hline spermathecae vs. testes $^{\mathrm{a}}$ & & 1 & 22.3 & 22.3 & 12.98 & 0.002 \\
\hline testis 1 vs. testis $2^{\mathrm{b}}$ & & 1 & 0.11 & 0.11 & 0.39 & 0.54 \\
\hline Male agexsource of sperm & 2 & & 1.99 & 1.99 & 1.00 & 0.38 \\
\hline Individual malexsource of sperm & 36 & & 36.0 & 2.0 & 1.36 & 0.08 \\
\hline individual male $\times$ (spermatheca vs. testes) & & 18 & 30.9 & 1.7 & 15.56 & $<0.001$ \\
\hline individual male $\times$ (testis 1 vs. testis 2 ) & & 18 & 5.1 & 0.28 & 1.93 & 0.01 \\
\hline Residual among images & 1080 & & 159.1 & 0.15 & & \\
\hline Total & 1139 & & 236.4 & 0.21 & & \\
\hline
\end{tabular}

${ }^{\text {aEffect }}$ of exposure to female tract. ${ }^{b}$ Variation between a male's testes.

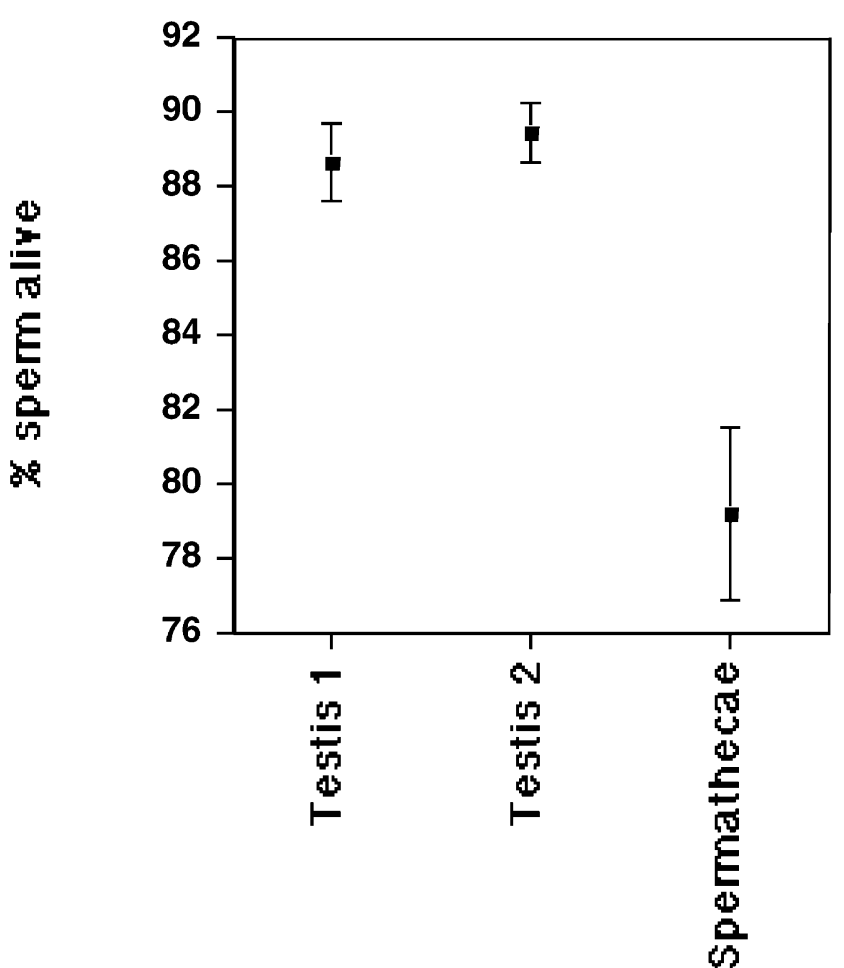

Fig. 2. Viability (mean \pm SE, $n=20$ mating pairs) of sperm from the spermathecae of singly-mated females and their mate's testes.

$\left.F_{1,18}=1.15, p=0.30\right)$, nor did male age, latency to $(4 \pm 4$ $\min )$ or duration of copulation $(36 \pm 10 \mathrm{~min}, n=20)$ predict viability (all $p \geq 0.15$ ).

In the control experiments, viability in samples from the testes examined directly after staining $(92.7 \pm 5.3 \%)$ did not differ significantly from viability in samples from the testes on which we applied pressure sufficient to break open a spermatheca $(92.0 \pm 7.9 \%$; paired $t$-test: $t=-0.23, \mathrm{df}=5, p=0.83)$. This indicates that the handling

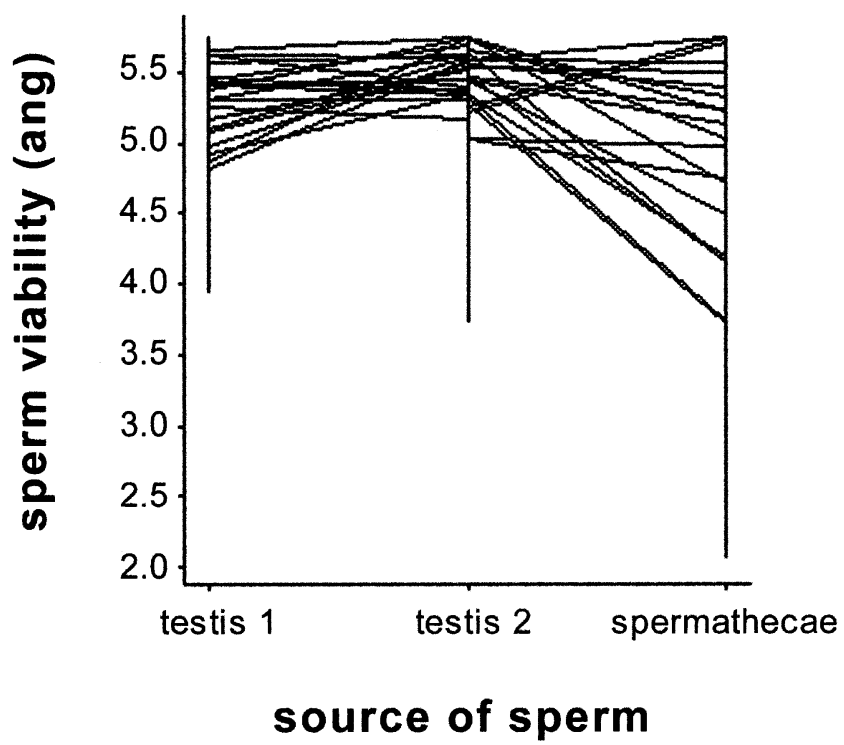

Fig. 3. Effect plot for sperm viability in the testes (1 and 2) of individual males and in the spermathecae of their mate. Lines connect values for each individual male. Sperm viability is angularly transformed $[(180 / \pi) \times \arcsin$ sqrt(proportion) $]$.

procedures to obtain sperm from the spermathecae vs. from testes did not affect sperm viability differently.

To identify whether parts of the female tract release substances which are toxic to sperm, we exposed sperm in vitro to isolated female reproductive tissues (female accessory glands, spermathecae, bursa copulatrix, and bursa copulatrix with female accessory glands), and to control tissues (male ejaculatory bulb, male flight muscle, female flight muscle, buffer only). Exposure to female reproductive vs. control tissues failed to reveal a spermicidal function of any of these extracts from parts of the female tract $\left(F_{7,154}=1.01, p=0.43,24\right.$ males, Tables 2 and 3). None of the linear contrasts comparing each 
Table 2

Analysis of variance for angularly-transformed in vitro sperm viability after exposure to female reproductive tissue (female accessory gland, bursa, spermathecae, bursa copulatrix with female accessory glands) vs. controls (male ejaculatory bulb, flight muscles of male and female, buffer only) in two experimental blocks

\begin{tabular}{|c|c|c|c|c|c|}
\hline Source of variation & $\mathrm{df}$ & ss & $\mathrm{ms}$ & $F_{\text {obs }}$ & $p\left(F>\left|F_{\text {obs }}\right|\right)$ \\
\hline Block & 1 & 619.2 & 619.2 & 0.42 & 0.52 \\
\hline Residual among males & 22 & 32224.2 & 1464.7 & 6.15 & \\
\hline Treatment & 7 & 1683.3 & 240.5 & 1.01 & 0.43 \\
\hline Treatment $\times$ block & 7 & 2549.2 & 364.2 & 1.53 & 0.16 \\
\hline $\begin{array}{l}\text { Residual among } \\
\text { treatments within } \\
\text { males }\end{array}$ & 154 & 36683.0 & 238.2 & 1.85 & \\
\hline $\begin{array}{l}\text { Residual among } \\
\text { images }\end{array}$ & $1529(7)$ & 196734.3 & 128.7 & & \\
\hline Total & $1720(7)$ & 270355.5 & & & \\
\hline
\end{tabular}

Table 3

Sperm viability (mean $\pm \mathrm{SD} ; \%)$ after in vitro exposure to (a) female reproductive tract extracts and (b) controls in two experimental blocks

\begin{tabular}{|c|c|c|c|c|}
\hline (a) & Accessory gland & Bursa copulatrix & Spermathecae & $\begin{array}{l}\text { Accessory gland and } \\
\text { bursa copulatrix }\end{array}$ \\
\hline Block 1 & $78.7 \pm 14.8$ & $83.0 \pm 11.5$ & $79.3 \pm 14.2$ & $80.5 \pm 14.4$ \\
\hline Block 2 & $82.9 \pm 15.1$ & $80.9 \pm 13.2$ & $82.3 \pm 14.7$ & $84.0 \pm 13.2$ \\
\hline (b) & Male muscle & Buffer only & Ejaculatory bulb & Female muscle \\
\hline Block 1 & $83.0 \pm 12.1$ & $82.0 \pm 13.1$ & $81.4 \pm 11.4$ & $82.9 \pm 12.6$ \\
\hline Block 2 & $85.7 \pm 13.4$ & $82.3 \pm 12.6$ & $83.0 \pm 14.9$ & $77.1 \pm 21.0$ \\
\hline
\end{tabular}

female reproductive tissue to the controls was significant (all $p>0.30$ ). Control tissues did not differ among each other $(p=0.16$, Table 2).

\section{Discussion}

The proportion of live sperm of a given male was significantly lower in the spermathecae (i.e. after mating and sperm storage) than it was in his testes after copulation. This reduction in sperm viability among stored sperm immediately after a single copulation indicates that sperm degradation already occurs during mating and sperm storage. In extreme cases sperm viability in the spermathecae can be up to $30 \%$ less than in the same male's testes, thus potentially putting a male at disadvantage compared to rival males competing for sperm storage and fertilization of the female's ova. By contrast, reduced sperm viability does not necessarily impact female fertility because after one mating females store over 1000 sperm cells (Otronen et al., 1997), which is sufficient to produce at least four fully fertile clutches (Parker, 1970), each of which consists of 55-65 eggs (Blanckenhorn, 2000). The control experiment confirmed that the lower sperm viability observed in vivo in the spermathecae cannot be ascribed to differences in the procedure to prepare sperm for examination. Also, sperm viability in the testes of virgin males (control experiment) was not lower than in the testes of singlymated males (in vivo experiment). This suggests that low sperm viability in the spermathecae cannot be due to "old" sperm having been transferred at mating. It is also unlikely that the sperm viability difference between testes and spermathecae could arise through preferential storage of dead sperm. Although sperm may be transported up the female tract independently of their motility (Simmons et al., 1999), under female musculature control (Hellriegel and Bernasconi, 2000), it would be counter-adaptive if storage were more efficient for dead sperm. Mechanical damage (sperm are tightly packed in the ducts during transfer and the duct surface is rough, Hosken et al., 1999) or failure in sperm and/or seminal fluid (loss of sperm function, failure in sperm motility, lack of sperm nutrients or necessary cofactors) may also provide an explanation, although selection to avoid such malfunctions clearly should be strong. In sum, the lower sperm viability in the spermathecae than in the testes immediately after mating is an intrinsic property of males (varying in sperm quality, motility, longevity) and/or is mediated by the female tract.

Importantly, we found significant and substantial variation among males in the proportion of live sperm reach- 
ing storage. This implies that a male's proportional contribution to the set of stored sperm when females mate multiply (e.g. 2-79\% for the second of two males, Hellriegel and Bernasconi, 2000) need not equal his chance of fertilization, because males can also differ in sperm viability and survival to storage. Recent work has focused on understanding sources of variance in male fertilization success (Lewis and Austad, 1990; Arnqvist and Danielsson, 1999) and sexual selection on sperm traits associated with fertilization ability (Keller and Reeve, 1995, Bernasconi and Keller, 2001). Future studies need to establish whether males vary heritably in sperm degradation resistance, and how this correlates with fertilization ability in competitive situations. Interestingly, in the present study variation among males in the proportion of live sperm reaching storage was independent of when males mated for the first time. That is, male age at first mating, and therefore possibly sperm age (Stockley, 1999), did not significantly affect either sperm viability in the male or sperm ability to resist degradation during transfer within the female tract. In $S$. stercoraria, males vary heritably in sperm length, although the causes of this variation are still unidentified (Ward, 2000b). It would thus be interesting to investigate whether variation among males in the sperm ability to resist degradation in the female tract is at least partly explained by variation in sperm morphology.

The mechanism of sperm death remains to be established, as none of the isolated female reproductive tissues (female accessory glands, spermathecae, bursa copulatrix, and bursa copulatrix with female accessory glands) to which we exposed sperm in vitro more strongly affected sperm mortality than control tissues (male ejaculatory bulb, male flight muscle, female flight muscle, buffer only, Table 3). In particular, in vitro the female accessory glands did not have the spermicidal function reported based on preliminary data in a previous study (Hosken et al., 2001). This lack of significance of the in vitro experiment does not allow us to determine whether reduced sperm viability in vivo after storage results from intrinsic male traits or from female influence (whereas a significant spermicidal effect of one or more of the female tract extracts would have strongly supported female influence). In most studies, lack of evidence does not imply lack of effect (Cohen, 1988). However, the statistical power of the in vitro experiment is very high, suggesting that one could conclude that none of the extracts of the female tract we investigated has a spermicidal function. Alternatively, lack of significance can be due to in vitro conditions that did not recreate the relevant physiological conditions, thus masking subtle treatment effects. Indeed, this may have been the case because viability values were generally low in vitro (Table 3).

Recently, female accessory gland were found to be larger in females mated to several rather than one male after 10 generations, suggesting a spermicidal function (Hosken et al., 2001). The present study, however, found no evidence in support of a spermicidal function, despite the high statistical power. Thus, it is possible that these glands, whose secretions are released during mating (Hosken and Ward, 1999), have a different function which is also related to the experimental regime of polyandry/monandry. For instance, lubrication to lower mating costs would be a function that is consistent with the design used by Hosken et al. (2001), which did not separate the two components of polyandry, i.e. mating with genetically different males and mating with more numerous males (see Bernasconi and Keller, 2001, for a design that separates these components). In sum, further work is needed to clarify the function of these glands.

In conclusion, we found significantly lower sperm viability in a female's spermathecae than in her mate's testes. Also, we found significant differences among males in the proportion of viable sperm that their mates store. Sperm mortality during mating and storage can result from intrinsic male properties (such as variation in sperm longevity) and/or from a spermicidal environment in the female tract. However, the mechanism remains to be identified. In vitro exposure to female reproductive accessory glands and other parts of the female tract failed to reveal a female spermicidal effect. Thus, to date, female muscular control of storage rates remains the only ascertained mechanism of differential sperm storage (Hellriegel and Bernasconi, 2000) in this species, confirming at least one of the mechanisms of paternity bias suggested by theory (Hellriegel and Ward, 1998). Indeed, differential storage across multiple spermathecae may be the most flexible mechanism of cryptic female choice at the time of egg-laying in a species where the relevant ecological conditions for optimal choice vary at a microgeographical scale (Ward, 2000a).

\section{Acknowledgements}

We thank Wolf Blanckenhorn, David Hosken, Eric Kubli, Bernhard Schmid and the referees for comments and discussion, Ruth Böhni, Dieter Burkhard, Jeannette Fanti, Gilbert Gradinger, Stefan Keller, Marc Kéry, Michel Nakano, Stefan Sommer and Anna Willimann for help, and Urs Greber for facilities. This work was supported by the Swiss Federal Program for Academic Recruitment (No. 409 GB, No. 167 BH) and Swiss NSF (No. 31-46861.96, PIW).

\section{References}

Andrès, J.A., Arnqvist, G., 2000. Genetic divergence of the seminal signal-receptor system in houseflies: the footprints of sexually 
antagonistic coevolution? Proceedings of the Royal Society B 268, 300-405.

Arnqvist, G., Danielsson, I., 1999. Copulatory behavior, genital morphology, and male fertilization success in water striders. Evolution 53, 147-156.

Arnqvist, G., Nilsson, T., 2000. The evolution of polyandry: multiple mating and female fitness in insects. Animal Behaviour 60, 145164.

Barnett, M., Telford, S.R., Tibbles, B.J., 1995. Female mediation of sperm competition in the millipede Alloporus uncinatus (Diplopoda: Spirostreptidae). Behavioral Ecology and Sociobiology 36, 413-419.

Bernasconi, G., Keller, L., 2001. Female polyandry affects their sons' reproductive success in the red flour beetle Tribolium castaneum. Journal of Evolutionary Biology 14, 186-193.

Birkhead, T.R., Møller, A.P., Sutherland, W.J., 1993. Why do females make it so difficult for males to fertilize their eggs? Journal of Theoretical Biology 161, 51-60.

Birkhead, T.R., 1998. Cryptic female choice: criteria for establishing female sperm choice. Evolution 52, 1212-1218.

Blanckenhorn, W.U., 2000. Temperature effects on egg size and their fitness consequences in the yellow dung fly. Evolutionary Ecology 14, 627-643.

Bloch Qazi, M.C., Herbeck, J.T., Lewis, S.M., 1996. Mechanisms of sperm transfer and storage in the red flour beetle (Coleoptera: Tenebrionidae). Annals of the Entomological Society of America 89, 892-897.

Chapman, T., Liddle, L., Kalb, J., Wolfner, M., Partridge, L., 1995. Cost of mating in Drosophila melanogaster females is mediated by male accessory gland products. Nature 373, 241-244.

Cohen, J., 1988. Statistical Power Analysis for the Behavioral Sciences. Erlbaum Ass, Hillsdale.

Degrugillier, M.E., 1985. In vitro release of housefly Musca domestica L. (Diptera: Muscidae) acrosomal material after treatments with secretions of female accessory-gland and micropyle cap substance. International Journal of Insect Morphology and Embryology 14, 381-391.

Eady, P.E., 1994. Sperm transfer and storage in relation to sperm competition in Callosobruchus maculatus. Behavioral Ecology and Sociobiology 35, 123-129.

Greeff, J.M., Parker, G.A., 2000. Spermicide by females: what should males do? Proceedings of the Royal Society B 267, 1759-1763.

Hellriegel, B., Ward, P.I., 1998. Complex female reproductive tract morphology: its possible use in postcopulatory female choice. Journal of Theoretical Biology 190, 179-186.

Hellriegel, B., Bernasconi, G., 2000. Female-mediated differential sperm storage in a fly with complex spermathecae, Scatophaga stercoraria. Animal Behaviour 59, 311-317.

Hosken, D.J., Ward, P.I., 1999. Female accessory reproductive gland activity in the yellow dung fly Scathophaga stercoraria (L.). Journal of Insect Physiology 45, 809-814.

Hosken, D.J., Meyer, E.P., Ward, P.I., 1999. Internal female reproductive anatomy and genitalic interactions during copula in the yellow dung fly, Scathophaga stercoraria. Canadian Journal of Zoology 77, 1975-1983.
Hosken, D.J., Garner, T.W.J., Ward, P.I., 2001. Sexual conflict selects for male and female reproductive characters. Current Biology 11, 489-493.

Johnstone, R.A., Keller, L., 2000. How males can gain by harming their mates: sexual conflict, seminal toxins and the cost of mating. American Naturalist 156, 368-377.

Jones, T.M., Balmford, A., Quinell, R.J., 2000. Adaptive female choice for middle-aged males in a lekking sandfly. Proceedings of the Royal Society B 267, 681-686.

Keller, L., Reeve, H.K., 1995. Why do females mate with multiple males? The sexually selected sperm hypothesis. Advances in the Study of Behaviour 24, 291-315.

Lewis, S.M., Austad, S.N., 1990. Sources of intraspecific variation in sperm precedence in red flour beetles. American Naturalist 13, $351-359$.

Michiels, N.K., Bakovski, B., 2000. Sperm trading in a hermaphroditic flatworm: reluctant fathers and sexy mothers. Animal Behaviour 59, 319-325.

Otronen, M.Ê., Siva-Jothy, M.T., 1991. The effect of postcopulatory male behaviour on ejaculate distribution within the female sperm storage organs of the fly, Dryomyza anilis (Diptera: Dryomyzidae). Behavioral Ecology and Sociobiology 29, 33-37.

Otronen, M., Reguera, M., Ward, P.I., 1997. Sperm storage in the yellow dung fly Scathophaga stercoraria: identifying the sperm of competing males in separate female spermathecae. Ethology 103, 844-854.

Parker, G.A., 1970. The reproductive behaviour and the nature of sexual selection in Scatophaga stercoraria. II. The fertilization rate and the spatial and temporal relationship of each sex around the site of mating and oviposition. Journal of Animal Ecology 39, 205-228.

Parker, G.A., 1990. Sperm competition games: raffles and roles. Proceedings of the Royal Society B 242, 120-126.

Pizzari, T., Birkhead, T.R., 2000. Female feral fowl eject sperm of subdominant males. Nature 405, 787-789.

Rice, W.R., 1996. Sexually antagonistic male adaptation triggered by experimental arrest of female evolution. Nature 381, 232-234.

Simmons, L.W., Parker, G.A., Stockley, P., 1999. Sperm displacement in the yellow dung fly Scatophaga stercoraria: an investigation of male and female processes. American Naturalist 153, 302-314.

Siva-Jothy, M.T., 2000. The young sperm gambit. Ecology Letters 3,172 .

Stockley, P., 1999. Sperm selection and genetic incompatibility: does relatedness of mates affect male success in sperm competition. Proceedings of the Royal Society B 266, 1663-1669.

Ward, P.I., 2000a. Cryptic female choice in the yellow dung fly Scathophaga stercoraria. Evolution 54, 1680-1686.

Ward, P.I., 2000b. Sperm length is heritable and sex-linked in the yellow dung fly Scathophaga stercoraria. Journal of Zoology 251, 349-353.

Ward, P.I., Simmons, L.W., 1991. Copula duration and testes size in the yellow dung fly, Scatophaga stercoraria L. The effects of diet, body size and mating history. Behavioural Ecology and Sociobiology $29,77-86$.

Wickler, W., 1985. Stepfathers in insects and their pseudo-parental investment. Zeitschrift für Tierpsychologie 69, 72-78. 